\title{
APROXIMACION AL PROBLEMA DE LA OFERTA Y DEMANDA DE TECNOLOGIA EN EL USO DE LOS SUELOS DE LA REGION DEL AMAZONAS
}

Rodríguez A chung Fernando (*)

\section{INTRODUCCION}

Cuando una persona observa por primera vez la selva baja, desde el avión, adquiere una visión panorámica de la Región, caracterizándola como una zona homogénea, casi plana, cubierta por una frondosa vegetación y serpenteada por numerosos cuerpos de agua. Esta percepción ha sido común en muchas personas que directa 0 indirectamente han estado ligados con la toma de decisiones en el desarrollo de la Región del A mazonas.

Dentro de esta concepción, las tierras de altura, como son las que cubren la mayor superficie de la región, han sido consideradas como prioritarias en las estrategias de desarrollo. Esto se refleja en las inversiones realizadas hasta el momento en la construcción de carreteras, tales como Iquitos $\mathrm{N}$ auta, J enaro Herrera-Colonia A ngamos, $\mathrm{N}$ apo - Putumayo y otras que se encuentran a nivel de estudios, como Iquitos - Costa N orte.

En cambio, las tierras inundables, donde la gran mayoría de la población rural sustenta sus estrategias productivas, han sido soslayadas 0 en su defecto han recibido un tratamiento no acorde con su importancia actual y potencial.

¿Cuáles son las características de estos dos ecosistemas?, ¿Dónde se asienta la población rural y dónde se localiza la producción agrícola?, ¿Cuál es la demanda de tecnología para el uso de estas áreas?, ¿Cuál ha sido la oferta de la investigación en el uso y manejo de los suelos de estos dos ecosistemas? Son preguntas que se pretende responder en el presente trabajo.

\section{ECOSISTEMAS TIPICOS}

El agua juega un rol importante en el paisaje amazónico (J unk, 1983), pues el río amazonas produce una descarga promedio anual de $175,000 \mathrm{~N}^{3} \mathrm{~S}^{-1}$ en el Océano A tlántico, lo que representa $1 / 5$ ó $1 / 6$ de todas las aguas de los ríos de los continentes (Sioli, 1984), y transporta cerca de $1.7 \times 106$ toneladas de sedimentos por año ala altura de la ciudad de Iquitos (M eade, 1979).

* Director General de Investigaciones de Suelos 
Desde este punto de vista, y tal como lo sugiere Meggers (1976) para toda la amazonía continental, en la Región del A mazonas, es posible reconocer dos ecosistemas do tamaño marcadamente contrastante y que difieren en su potencial de subsistencia: la basta "Tierra firme", en donde los recursos están muy dispersos pero continuamente disponibles y la estrecha llanura de inundación, denominada "várzea" en el Brasil, en donde alternan la escasez y la abundancia, según suba o baje el nivel del río.

El ecosistema de "Tierra firme" o de "Tierras de altura" se localiza en las terrazas altas, lomadas y colinas, no soportan inundación y se encuentran cubiertas por una floresta tropical frondosa. Constituye un ecosistema cerrado, donde el ciclo de la mayor parte de nutrientes se da fundamentalmente entre el suelo y la biomasa que soporta. Tiene como sustento geológico a formaciones del plio - pleistoceno y cubren una superficie aproximada de 31.7 millones de hectáreas, que representa el $92 \%$ de las tierras de la Región; son de difícil acceso y de vocación predominantemente forestal.

En cambio, la "várzea" o tierras inundables, que se localiza en islas, complejo de orillares y en terrazas bajas, tienen origen reciente, holocénico, y constituye un ecosistema abierto. Según J unk (1983), la várzea es comparable a un gran transformador biológico, pues, estas áreas son anualmente fertilizadas por la inundación, posteriormente devuelven al río una cantidad de nutrientes equivalentes a aquellos recibidos en forma inorgánica, como sales disueltas y sedimentos. En la várzea, ellos son transformados parcialmente por medio de la energía solar en materia orgánica y devuelto al río en forma de plantas acuáticas, detritos orgánicos, troncos de árboles, sustancias orgánicas disueltas, etc., mediante este proceso dinámico estas áreas logran tener un equilibrio ecológico. Se estima que este ecosistema cubre 2.7 millones de hectáreas que representa el $8 \%$ de las tierras de la región. Son las más accesibles y en éste se localizan los suelos más fértiles con vocación para cultivos en limpio.

Por otro lado, en términos generales, la Región del Amazonas posee mas vocación forestal, pues el $80.14 \%$ de su territorio pertenece a esta capacidad de uso mayor (ver Cuadro N2 1); sin embargo, en cifras absolutas, existe 540,000 Ha. para cultivos en limpio, ubicados mayormente en áreas inundables, 607,000 Ha. para cultivos permanentes, de los cuales una parte significativa son adyacentes 0 accesibles a los principales ríos de la Región. A mbos tipos de tierra representan el 15\% del potencial agrícola del país. El uso de estas tierras presentan serias limitaciones, ya sea por inundación, en el caso de los suelos aluviales recientes, o por deficiencias químicas en las tierras de altura (deficiencia de nitrógeno y fósforo, bajas reservas de potasio y magnesio, toxicidad de aluminio, etc.). Con tecnología es posible ampliar significativamente los límites estimados por ONERN sobre el potencial para fines agrícolas. 


\section{CUADRO №1 \\ CAPACIDAD DE USO MAYOR DE LAS TIERRAS DE LA REGION DEL AMAZONAS}

(En miles de Hectáreas)

\begin{tabular}{|l|r|r|r|r|}
\hline \multicolumn{1}{|c|}{$\begin{array}{c}\text { Capacidad } \\
\text { de U so }\end{array}$} & \multicolumn{2}{c|}{$\begin{array}{r}\text { Región A mazonas } \\
\text { Superficie }\end{array}$} & \multicolumn{1}{c|}{ Perú } & \multicolumn{1}{c|}{$\begin{array}{c}\text { de } \\
\text { Región A maz. }\end{array}$} \\
\hline Cultivos en limpio & 540 & 1.6 & 4,900 & 11 \\
Cultivos perennes & 607 & 1.8 & 2,700 & 22 \\
Pastos & 2,229 & 6.5 & 17,900 & 12 \\
Forestales & 27,605 & 80.1 & 48,700 & 57 \\
Protección & 3,465 & 10.0 & 54,300 & 6 \\
\hline TOTAL & 34,446 & 100.0 & 128,500 & 27 \\
\hline
\end{tabular}

Fuente ONERN (1982)

\section{POBLACION Y PRODUCCION AGRICOLA}

La población de la Región del A mazonas, estimada para 1989, fue de 638,000 habitantes, representando sólo el $2.9 \%$ del total nacional. U na característica fundamental de la ocupación del territorio es que la población se concentra en áreas adyacentes a los ríos, lo que refleja el papel importante que desempeñan los cuerpos de agua en el desarrollo económico de la región, los ríos constituyen el principal medio de transporte intrarregional, no sólo de pasajeros sino también de carga, así como el hábitat donde viven los peces, que es la principal fuente de proteína que consume la población.

L os grandes centros poblados, como Iquitos, $\mathrm{Y}$ urimaguas, Requena y $\mathrm{N}$ auta, se ubican en tierras de altura, que afloran al borde de los ríos, mientras que gran parte de los pequeños poblados del área rural se encuentran en forma dispersa en áreas inundables, principalmente en 'restingas'.

Los ríos A mazonas, U cayali, Huallaga y Marañón, son los más densamente poblados, pues cerca del $86 \%$ de la población se localiza en estos ríos (V er Cuadro № 2).

Por otro lado, desde el punto de vista económico, el agro es poco significativo, pues en 1988 sólo representó el 9\% del PBI de la Región del A mazonas y el 3.3\% del PBI agrícola nacional (Cuanto S.A., 1990). A simismo, sólo cuatro (4) cultivos dominan el escenario agrícola de la Región: arroz, maíz, yuca y plátano, estos cubrieron cerca del 92\% de la superficie sembrada en 1989 (V er Cuadro № 3).

Se estima que cerca del $80 \%$ de la superficie sembrada con productos agrícolas se localiza en áreas inundables: el arroz en barrial; maíz, plátano, yuca y urena en restinga; caupíy maníen playas, principalmente. En cambio, sólo el $20 \%$ de la superficie se ubica en tierras de altura: arroz en secano en la zona de Y urimaguas, parte del plátano y yuca, cultivo de caña y cítricos, entre los más importantes. 
Es relevante que el $95 \%$ de la superficie sembrada en 1989, estón ubicadas en áreas adyacentes a los ríos de origen andino (Ver Cuadro $\mathrm{N}$ ㅇ 3), debido a que los suelos de mayor fertilidad natural se localizan en estas zonas. Estos aspectos inducen a pensar que existe una lógica del agricultor en ubicar sus sembrios en las zonas que presentan mayor fertilidad de suelos, pues priorizan las tierras bajas inundables y los ríos que depositan sedimentos ricos en nutrientes. Sin embargo, si se considera sólo el potencial de suelos para cultivos en limpio, la superficie sembrada sólo representaría el 13\% de dicho potencial, teniendo por lo tanto posibilidad de ampliar significativamente la actual frontera agrícola regional.

\section{CUADRO № 2}

DISTRIBUCION DE LA POBLACION EN LOS PRINCIPALES RIOS DE LA REGION DEL AMAZONAS

1981

\begin{tabular}{|l|r|r|r|r|r|r|}
\hline \multicolumn{1}{|c|}{ Ríos } & \multicolumn{1}{c|}{$\begin{array}{c}\text { Urbano } \\
\text { Habitant. }\end{array}$} & \multicolumn{1}{c|}{$\%$} & $\begin{array}{c}\text { Rural } \\
\text { Habitant }\end{array}$ & \multicolumn{1}{c|}{$\%$} & $\begin{array}{c}\text { Total } \\
\text { H abitant. }\end{array}$ & \multicolumn{1}{c|}{$\%$} \\
\hline A mazonas & 186,659 & 73.1 & 71,291 & 31.3 & 257,950 & 53.4 \\
U cayali & 26,069 & 10.2 & 51,316 & 22.6 & 77,385 & 16.0 \\
Huallaga & 27,945 & 11.0 & 20,747 & 9.1 & 48,692 & 10.1 \\
M arañon & 5,981 & 2.3 & 26,145 & 11.5 & 32,126 & 6.7 \\
Otros & 8,636 & 3.4 & 58,140 & 25.5 & 66,676 & 13.8 \\
\hline TOTAL & 255,290 & 100.0 & 227,539 & 100.0 & 482,829 & 100.0 \\
\hline
\end{tabular}

Fuente: INE., Censo Nacional de Población y Vivienda 1981.

\section{CUADRO № 3}

\section{DISTRIBUCION DE LA SUPERFICIE SEMBRADA DE PRODUCTOS AGRICOLAS EN LOS PRINCIPALES RIOS DE LA REGION DEL AMAZONAS} (En H ectáreas) 1989

\begin{tabular}{|c|r|r|r|r|r|r|r|}
\hline Ríos & \multicolumn{1}{|c|}{ Arroz } & \multicolumn{1}{c|}{ M aíz } & Y uca & Plátano & Otros & \multicolumn{2}{|c|}{ Total } \\
\cline { 6 - 8 } & & & & & & Ha. & \multicolumn{1}{c|}{$\%$} \\
\hline A mazonas & 5,500 & 2,083 & 3,724 & 1,578 & 2,872 & 15,757 & 21.9 \\
U cayali & 6,972 & 12,300 & 2,954 & 1,836 & 1,087 & 25,149 & 34.9 \\
Huallaga & 5,274 & 6,900 & 1,959 & 974 & 268 & 15,375 & 21.3 \\
M arañon & 5,959 & 2,478 & 1,344 & 994 & 1,221 & 11,996 & 16.7 \\
Otros & 995 & 1,739 & 519 & 218 & 271 & 3,742 & 5.2 \\
\hline TOTAL & 24,700 & 25,500 & 10,500 & 5,600 & 5,719 & 72,019 & 100.0 \\
\hline
\end{tabular}

Fuente: Dirección Regional de A gricultura de L oreto.

(*)Incluye urena, caña de azúcar, maní, limón y naranja 


\section{OFERTA Y DEMANDA DE TENOLOGIA EN EL USO DE LOS SUELOS}

\section{- DE MANDA}

En términos generales, la estrategia productiva del poblador rural de la Región del Amazonas se sustenta en el uso de los diversos biotipos existentes, tanto en ecosistema inundables como en ecosistemas de tierras de altura, siendo la primera de ellas el eje central de esta estrategia, pues la principal fuente de proteína vegetal y animal procede de los ecosistemas inundables. En cambio, los ecosistemas de tierra de altura son mas fuentes de carbohidratos y desempeñan un papel complementario de gran importancia en las épocas de inundación.

En ta sentido, se identifica una demanda del poblador ribereño por mejorar sus tecnologías para el uso y manejo de los suelos ubicados en los diversos biotipos adyacentes a los principales ríos, que se denominará demanda real.

Por otro lado, existe otro tipo de demanda que aquí se llamará demanda inducida y que se refiere a nuevas necesidades creadas por las propias instituciones de desarrollo, a través de los proyectos de construcción de carreteras, con el afán de incorporar "tierras agrícolas" y establecer programas de colonización; es decir, inducir a la población ribereña para trasladarse a tierras de altura y, en algunos casos, con alguna justificación "geopolítica", para incorporar las zonas fronterizas. Este tipo de demanda está orientada a buscar nuevas opciones tecnológicas para el uso de estos suelos, pues con una mayor presión demográfica, los sistemas tradicionales de 'roza y quema se tornan peligrosos de una degradación ecológica.

\section{- OFERTA}

Tres tipos de agentes son los generadores de oferta de tecnología, la población nativa y ribereña, los colonos migrantes y las instituciones de investigación y/o desarrollo.

A quí cabe señalar que en la Región del A mazonas, no se ha registrado grandes avances tecnológicos en el campo agrícola, tal como ha sido en otras regiones del país. Sobre el particular, M acera (1990), manifiesta que en el Perú pre-Inca, se han generado diversas tecnologías, tales como irrigaciones por canales, andenes, chacras inundadas, islas flotantes, huaru huaru, chaquitaclla, uso de pescado como abono, etc. Lo más relevante, generado o adaptado por los nativos de esta región, es el sistema de "rozo y quema", que se caracteriza, según Dourojeanni (1982), por constituir un sistema agrícola basado en la rotación del campo mucho más que en la del cultivo, aprovechando mucha tierra disponible con suelos de escasa fertilidad natural, alternando períodos cortos de cosecha (2-4 años). El principio básico se sustenta en el aprovechamiento de los nutrientes contenidos en la materia orgánica que se encuentra en tránsito en el suelo, como parte del ciclo natural, y mediante la liberación de estos durante la quema de la biomasa. 
¿A qué se debe esta situación?, ¿Porqué el poblador de esta región no ha generado mas tecnologías para el uso de estos suelos? A continuación se pretende dar respuestas a estas interrogantes.

Según San Román (1975), el hombre de la región no fue agricultor ni ganadero, tal como se desprende de los primeros testimonios escritos a partir de la llegada de Francisco de Orellana (1542). Pues sólo existía pequeñas chacras de yuca, plátano y, en menor escala, maíz y maní, que servían como complemento de sus dietas y, sobre todo, para la preparación del masato y de la chicha, que eran utilizados en sus fiestas. Estos trabajos eran realizados por lo general por la mujer.

En cambio, la base económica del poblador nativo ha sido la caza, la pesca y la recolección. Esto es explicable, en la medida que los diversos ecosistemas de la región ofrecían una diversidad de productos, tanto en el tiempo como en el espacio, que satisfacía la demanda de la pequeña y dispersa población existente en la región. Por consiguiente, el mayor esfuerzo de esta población no ha sido en conocer y manejar los suelos que son el sustento de la agricultura, sino más bien al bosque en su conjunto.

Recién, a partir de 1600, con la llegada de los misioneros jesuitas, se busca un nuevo estilo de producción, acorde con la nueva estrategia de ocupación del espacio (formación de centros de mayor densidad poblacional), donde la agricultura adquiere una relativa importancia, se introduce nuevos cultivos, como naranjos, limones, coles, lechugas, etc., así como se promueve la cría de gallinas, patos, cerdos y vacas. A parecen las chacras comunales, llamadas de la misión, cuyos productos estaban orientados a la alimentación de los menores de edad que vivían en la misión, así como de los necesitados y transeúntes (San Román, 1975).

Posteriormente, el sistema de "roza y quema fue modificado, encontrándose diversas variantes, como el sistema agroforestal de Tamshiyacu, que tiene como cultivo central al umarí (Poraqueiba sericea), (Padoch et al 1990), purmas manejadas de los Boras (Denevan y Treacy 1990) y huertos domésticos, etc. (Bidegaray y Rhoades, 1986). En términos generales, estos sistemas se caracterizan por la siembra y conservación de diversas especies agrícolas o forestales en un espacio dado y, en algunos casos, asociadas a animales menores (como en los huertos domésticos), simulando la estructura y funcionamiento de los eco sistemas naturales.

Con excepción de la agroforestería de Tamshiyacu, que está orientada al mercado de Iquitos y que genera excedentes superiores a otros sistemas similares de esta zona (Padoch et al. 1990), el sistema de "rozo y quema", incluyendo sus diversas variantes, se desarrollan dentro del marco de una economía de subsistencia.

Por el año de 1981, algunos campesinos de las zonas de Cajamarca, Rioja y Lambayeque migraron a Yurimaguas, trayendo consigo la tecnología de arroz bajo riego, que fue difundiéndose progresivamente en el área de influencia de esta ciudad (Bidegaray et al 1989?). Esta quizá ha sido la única experiencia de introducción de tecnología para el manejo de suelos, procedente de otra región del país. Lamentablemente, por los problemas derivados en la comercialización del arroz, mucho de estos colonos se han visto obligados a cambiar parte de sus cultivos por el sembrío de la coca, producto que probablemente genera menos excedentes, pero con 
comercialización asegurada. A simismo, cabe mencionar la introducción de tecnología de manejo de suelos de altura para el cultivo de palma aceitera en el río M aniti (Iquitos), que implica al control de la nutrición de los árboles, aplicación de fertilizantes y cobertura del suelo con kudzú. Esta tecnología, desarrollada por una institución francesa (R.I.H.O.) fue primeramente adaptada a las condiciones de la amazonía peruana en las plantaciones de Tocache (San Martín). Desde inicios de la década del 70, diversas instituciones nacionales y regional s de investigación, han desarrollado estudios orientados a determinar la potencialidad y limitaciones d. los suelos de la región, así como a generar tecnologías para su manejo.

La Oficina Nacional de Evaluación de Recursos N aturales (ONERN), ha sido la institución que más estudios de inventario y evaluación de suelos ha realizado en la Región del A mazonas. Otras instituciones, como CEPIP de la Universidad Agraria de L a Molina, M inisterio de Agricultura y el IIAP, también realizaron algunos estudios puntuales en este campo. Sin embargo, la constante es que gran parte de estos han sido realizados a nivel macro, que sólo permiten dar una visión general de los suelos para una planificación de nivel regional. Estudios detallados o de caracterización a nivel micro son poco relevantes. Sólo el $0.44 \%$ y $0.67 \%$ del territorio de la Región del A mazonas han sido estudiados a nivel de detalle y semidetallado, respectivamente (V er C uadro $N \cong 4$ ).

Generalmente, ha sido muy poco el uso que se le ha dado a esta información, en algunos casos hasta parece que se ha soslayado estos estudios, tal como el realizado por el $\mathrm{M}$ inisterio de A gricultura para la carretera I quitos

- Nauta, donde se revela que las áreas por incorporarse son mayormente de vocación forestal y las pocas áreas agrícolas ya habían sido incorporados con las antiguas carreteras existentes en I quitos.

En cuanto a tecnologías para el uso y manejo de los suelos, gran parte del esfuerzo se ha concentrado en los problemas de tierras de altura, destacando los trabajos realizados por el INIAA, en convenio con la U niversidad Estatal de Carolina del Norte, en la zona de Y urimaguas. En esta estación, desde 1970 se han desarrollados estudios para encontrar opciones tecnológicas para el manejo de los suelos ácidos de esta parte tropical. La mayor concentración de infraestructura y de personal altamente calificado que se ha registrado en toda la amazonía peruana, se reporta en Y urimaguas. Cerca de 20 años de investigación permanente, han generado conocimientos y opciones tecnológicas para el uso de estos suelos. Destacan las opciones siguientes:

- A rroz bajo riego: en restingas y otras zonas con suelos aluviales de mediana a alta fertilidad, pero no inundables, con capacidad de producir dos cosechas al año, con un promedio de $5 \mathrm{Ton} / \mathrm{Ha}$., cada una a nivel de agricultor. 
-Rotación intensiva de cultivos: en base a arroz, maíz, soya y maní, utilizando fertilizantes y otros insumos. Esta opción, por sus requerimientos financieros, de infraestructura y de conocimientos por parte del agricultor, no ha podido ser promovida en la Región. Como al ternativa se está investigando nuevas opciones con bajos insumos, bajo la concepción de "adaptar las plantas a las limitaciones del suelo", en vez de "modificar el suelo para cubrir las necesidades de las plantas".

- Cultivos perennes y agroforestales: Dentro de esta opción, destaca como lo más promisorio el intercalar cultivos perennes de alto valor unitario, como el pijuayo, con cultivos anuales. En la zona de lquitos, en el marco del Convenio del Gobierno Regional del Amazonas con el Instituto de Cooperación IberoAmericana de España, se está promoviendo a nivel de agricultor los resultados de esta opción, utilizando pijuayo y arazá en sistemas agroforestales.

- Pasturas con leguminosas, utilizando fertilizantes: también es considerado como una opción para el manejo de los suelos ácidos. Falta determinar su viabilidad socioeconómica a nivel de productor.

A simismo, el IIAP en estos últimos años ha iniciado trabajos en sistemas agroforestales en Jenaro Herrera, con resultados preliminares aún no transferibles al agricultor. Por otro lado, la UNAP viene desarrollando desde hace más de 10 años sistemas agroforestales, en base a la experiencia de nativos del Ampiyacu, pero, lamentablemente hasta la fecha, no se cuenta con ninguna información sobre este proyecto. Completa este marco, estudios desarrollados por el INIAA (Iquitos) para la selección de variedades de arroz y caupí para suelos de áreas inundables; recién en estos últimos años se reporta mayor inquietud para conocer estas áreas, se aprecia así trabajos de Hoag (1985), sobre la variabilidad de la fertilidad de los suelos ubicados en diversos ríos de la

\section{CUADRO № 4}

\section{SUPERFICIE CUBIERTA CON ESTUDIOS DE INVENTARIO Y EVALUACION DE SUELOS EN LA REGION DEL AMAZONAS}

\begin{tabular}{|l|c|c|}
\hline \multicolumn{1}{|c|}{ Nivel del Estudio } & Superficie & Cobertura \\
& Estudiada $(\mathrm{Ha})$ & Regional $(\%)$ \\
\hline Exploratorio & 215,800 & 0.63 \\
Reconocimiento & $7,330,474$ & 21.27 \\
Semidetallado & 229,414 & 0.67 \\
Detallado & 152,356 & 0.44 \\
\hline Total & 7 '928,044 & 23.01 \\
\hline
\end{tabular}


Región; del IIAP (Rodríguez, et al., 1989), sobre caracterización de suelos ubicados en un complejo de orillares del río Amazonas; del Convenio IIAP UNAP-INIAA y U niversidad Carolina del $\mathrm{N}$ orte, sobre fertilidad natural de suelos aluviales entre Iquitos y Nauta; y del proyecto Landsystem - Gobierno Regional del A mazonas, sobre estudios de sorgo y soya en restinga baja; y estudios de camu-camu en áreas inundables, del IV ITA -IN IA A (Iquitos); éstos son al gunos ejemplos de como va adquiriendo importancia el estudio de estas áreas.

Como se podrá observar, el mayor esfuerzo en conocer y generar tecnologías para el uso y manejo de los suelos ha estado orientado a tierras de altura. Esto revela una oferta limitada que no cubre con las expectativas globales de la demanda real; se aprecia, asimismo, que ha sido escaso el avance en áreas inundables, del IVITA-INIAA (Iquitos); éstos son algunos ejemplos de como va adquiriendo importancia el estudio de estas áreas.

Como se podrá observar, el mayor esfuerzo en conocer y generar tecnologías para el uso y manejo de los suelos ha estado orientado a tierras de altura. Esto revela una oferta limitad que no cubre con las expectativas globales de la demanda real; se aprecia, asimismo, que ha sido escaso el avance en áreas inundables, donde precisamente el poblador ribereño centra su estrategia productiva.

\section{BIBLIOGRAFIA}

\section{BIDEGARAY, P. y R. RHOADES}

1986 LoS agricultores de Y urimaguas: Uso de la tierra y estrategias de cultivo en la selva peruana.

Documentos 10, CIPA, Lima.

CUANTO S.A.

1990 ¿Cuánto? Un balance del proceso de desarrollo.

IPAE, Lima.

DENEVAN W. y J. TREACY

990 Purmas jóvenes manejadas en Brillo Nuevo. En: A groforestería tradicional en la amazonía peruana.

Documento 11. CIPA, Lima.

DOUROJEANNI, M.

1982 Recursos Naturales y Desarrollo en A mérica L atina y el Caribe. U. de Lima, Lima. 
HOAG, R.

1985 Characterization of soil on floodplains of tributaries flowing into the Amazon River in Perú.

Tésis Doctoral en preparación, NCSU.

INE

1983 Censo Nacional de Población y Vivienda 1981. Lima.

JUNK, J.

1983 As aguas de region amazonia. In: A mazonia: Desenvolvimiento, integración e ecología. Editora Brasilense S. A., Brasil.

MACERA, P

1990 Historia de los Incas. Editorial Bruño, Lima.

MEADE, R., et al

1979 Transporte de sedimentos no río A mazonas: Acta A mazónica 9 [3. 543 - 547, Brasil.

MEGGERS, B.

1976 A mazonia un paraiso ilusorio. Siglo XXI Editores S. A., M éxico.

ONERN

1982 Clasificación de las Tierras del Perú. Lima.

PADOCH, C., et al

1990 La agroforestería orientada hacia el mercado en Tamshiyacu. En: A groforestería tradicional en la amazonia peruana. Documento 11, CIPA, Lima.

RODRIGUEZ, F., G. PAREDES y J. RAMIREZ

1989 Algunas características físicas y químicas de estratos sedimentarios de áreas aluviales recientes en el río A mazonas. IIAP, Iquitos.

SA N ROMAN, J.

1975 Estudio socio económico de los ríos A mazonas y N apo. CETA, Iquitos.

SANCHEZ, P., y J. BENITES

1983 Opciones tecnológicas para el manejo racional de suelos en la selva peruana. Serie Separatas N o. 6, IN IPA, Lima. 